\title{
The Histological Study of Orthodontic Force on the Periodontal Tissues Regenerated by Nano Bioceramics in Beagle Dogs
}

\author{
Lan-lei WANG ${ }^{1}$, He-ying $\mathrm{HOU}^{2}$, Sheng-yan $\mathrm{YU}^{1}$, Ai-zhong \\ GUAN $^{3, a, *}$ and Yun-mao LIAO ${ }^{4}$
}

${ }^{1}$ Affiliated Hospital of Shandong University of Traditional Chinese Medicine, China.

${ }^{2}$ Department of stomatology, Zaozhuang Municipal Hospital, China.

${ }^{3}$ Linyi People's Hospital, Linyi, Shandong, China.

${ }^{4}$ State Key Laboratory of Oral Diseases, Sichuan University, Chengdu, China.

a406975817@163.com

${ }^{*}$ Corresponding author

Keywords: Nano Biphasic Calcium Phosphate, Orthodontic Force, Tooth Movement.

\begin{abstract}
The aim of the experiment was to study the histological reaction of orthodontic force on the periodontal tissues regenerated by nano biphasic calcium phosphate (NBCP) bioceramics in beagle dogs. Two third incisors were randomly selected and operated as experimental group. The contralateral third incisors of the same jaw were done nothing as control. Alveolar bone defects were surgically created at the labial aspects of the third incisors in two beagle dogs. After root conditioning with ethylenediaminetetraacetate (EDTA), NBCP was filled in the defects. At 24 weeks after implantation the third incisors were moved labially with the adjacent canines for anchorage. Four weeks later dogs were killed. Specimens were processed and stained with hematoxylin and eosin (HE). The results revealed that there was no significant difference in the histological reaction induced by orthodontic tooth movement between the two groups.
\end{abstract}

\section{Introduction}

In clinical practice, many patients with damaged periodontium have the demands for orthodontic treatment. Regeneration of lost periodontium is the focus of periodontal and orthodontic therapy. The complete and predictable restoration of the periodontium following trauma or infection remains a critical objective in periodontics. To date, various treatment modalities such as implantation of bone graft materials, guided tissue regeneration, growth factors and combination of these approaches, have been employed in clinical practice. However, the clinical results obtained with these methods vary widely and are often unpredictable [1-4]. Bone replacement grafts are widely used in the treatment of periodontal osseous defects. But it is generally accepted nowadays that bone grafting materials are defect fillers and can induce periodontal regeneration unpredictably [5-7].

With osteoconduction, biocompatibility and bioactivity properties, nano porousbiphasic calcium phosphate (NBCP) has already been proven its efficacy on periondontal regeneration in our former studies [8-10]. The aim of the experiment was to study the histological reaction of orthodontic force on the periodontal tissues regenerated by $\mathrm{NBCP}$ in beagle dogs. 


\section{Material and Method}

The operations were performed on two 1.5-year-old male beagle dogs (body weight $10.1 \pm 0.8 \mathrm{~kg}$ ). The study protocol was approved by the Animal Ethical Committee for Animal Research of Sichuan University (Chengdu, China). NBCP were supplied by the Material Lab of State Key Laboratory of Oral Diseases, Sichuan University (Fig.1).

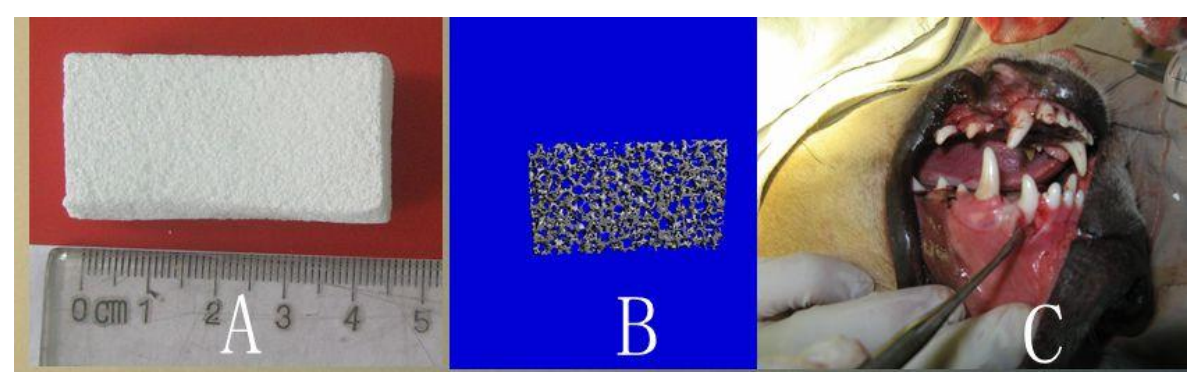

Fig.1 (a) The aspect of NBCP sample; (b) reconstruction transect image of NBCP by $\mu$ CT; (c) the alveolar bone block about $3 \mathrm{~mm}$ wide, $4 \mathrm{~mm}$ high and deep into the root surface was removed from the labial aspect of the third incisors.

All surgical procedures were performed by one experienced surgeon. General anaesthesia was performed with an intravenous injection of $3 \%$ pentobarbital sodium (Shanghai, China, $30 \mathrm{mg} / \mathrm{kg}$ ). 2 third incisors were randomly chosen and operated as experimental group. The contralateral of the same jaw was done nothing as control. Following mesial vertical incisions, a full-thickness mucoperiosteal flap was raised to expose the labial aspect of the third incisors, where a rectangular piece of the bone, about $4 \mathrm{~mm}$ high, $3 \mathrm{~mm}$ wide and deep into the root surface, was removed with an osteotome. Reference notches indicating the bottom of the defect were made on the respective root surfaces with a high-speed handpiece under saline irrigation (fig.1c). Periodontal ligaments were scaled thoroughly. All root surfaces in defects had been conditioned with EDTA (MD-ChelCreamTM, Meta Biomed Co., Ltd, Korea) for two minutes. The surgical sites were rinsed thoroughly with sterile saline. NBCP was trimmed into granules of about $0.5-1 \mathrm{~mm}^{3}$ and immersed into sterile saline. Then it was filled in the defects. Finally, the flaps were repositioned and sutured.

After the operation, the animals received an antibiotic (Penicillin G, 800,000 IU) administered intramuscularly. Sutures were removed 2 weeks later. Chemical plaque control $(0.2 \%$ chlorhexidine rinsing and then iodine glycerin smearing) was performed once a day for 2 weeks and then reduced to twice a week until the animals were sacrificed. During the experiment, the dogs were fed with soft-food diet to minimize mechanical trauma to the flaps.

The third incisors were moved labially with the adjacent canines for anchorage at 24 weeks after implantation. Two weeks later dogs were killed. Labial arch was made with the Australian wire of 0.016 inches, stop loops were made in the medial bands. In order to avoid wires slipping, helical loops were made in the wires to place orthodontic ligature wire (fig. 2). The orthodontic force was $100 \mathrm{~g}$.

At four weeks, two dogs were sacrificed. The experimental teeth together with surrounding tissues were harvested. For hard tissue histology, the specimens were fixed in $4 \%$ paraformaldehyde, then put $37^{\circ} \mathrm{C}$ thermostat, EDTA decalcification, paraffin embedded, sliced. $7 \mu \mathrm{m}$ histological sections were cut in the labiolingual direction, parallel to the long axes of the teeth with a microtome (Leica SM 2500E, Germany). The sections were stained with hematoxylin and eosin before examination with a light 
microscope (Leica DMR). The images were captured by a digital camera connected to that light microscope.

\section{Placement of Appliances}

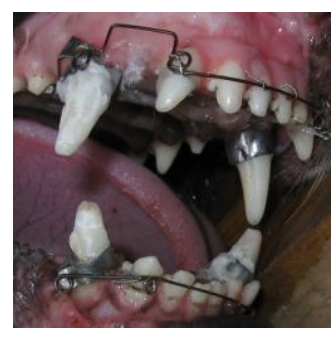

Fig.2. Placement of appliances

\section{Experimental Results}

All surgeries went well and the clinical incision healing was uneventful in all the animals. In all cases, no complications such as inflammation and exposure of NBCP were observed after operation.

The histological reaction of the regenerated periodontal tissues induced by experimental tooth movement was examined in 2 beagle dogs. Because movement of labial inclination was used on the drifting tooth in the experiment and the zone of operation was on the periodontal tissue of the tooth root at labial side, the observational region showed mainly tension on periodontal ligament. There is no difference between newly formed periodontium and the normal periodontal tissues.
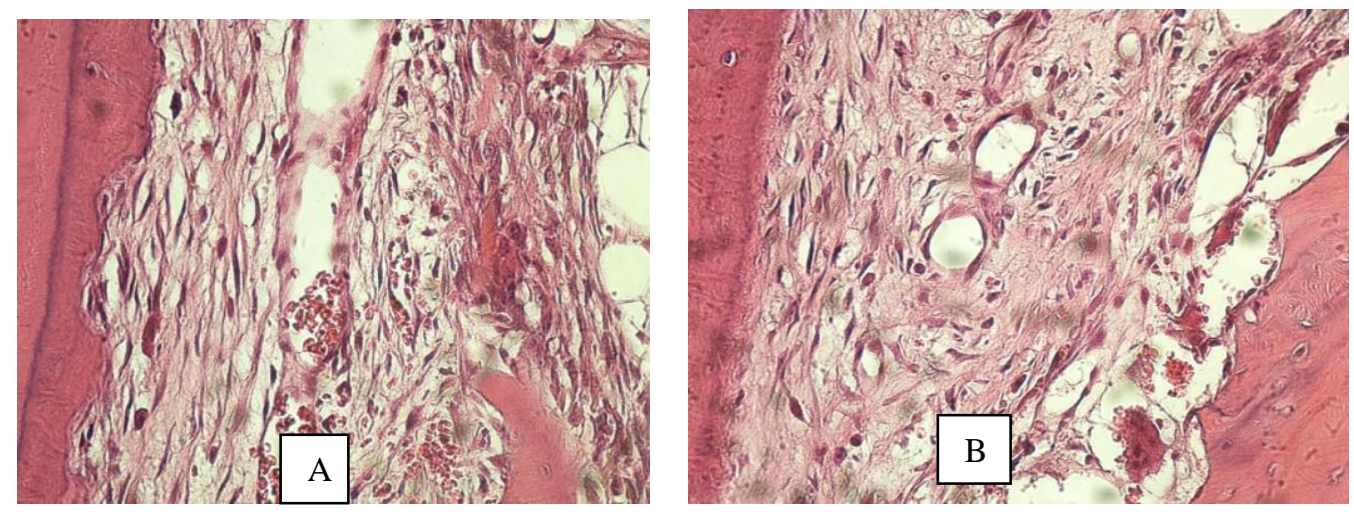

Fig. 3. Histological change 4 weeks after orthodontic movement $(\mathrm{HE} \times 400)$; A: experimental group, B: control group

\section{Discussion and Conclusion}

It is proved BCP has good efficacy on bone regeneration as a bone substitute in different clinical applications. With the small-size effect and the surface effect, nano-ceramic material shows different characteristics from traditional ceramic. Research has shown that microstructure and sub-microstructure on the surface of tissue engineering scaffold have a great influence on cell adhesion and growth [8,9]. Its macropores provide space for bone ingrowths and its micropores allow the transportation of body fluid, which leads to angiogenesis and helps tissue regeneration. Increase of its specific surface area enhances its degradation greatly and accelerates interface bonding reaction $[10,11]$. 
As for optimal bone regeneration, the degradation rate of BCP should equalize the rate of bone ingrowths. The biodegradation of BCP depends on many factors, such as HA/ $\beta$-TCP ratio, porosity, degree of bony contact, specific surface, type of bone, species of animal, age, sex etc[12]. Ideally, when any factor varies, BCP should be readjusted to accommodate the specific implantation site consequently. By adapting the proportions of $\mathrm{HA} / \beta-\mathrm{TCP}$ and the pores structure, it is possible to control biodegradation of BCP. However, it is still a challenge to optimize the appropriate porous structure and the composition of $\mathrm{BCP}$ to accommodate bone-specific implantation sites.

An important factor affecting degradation of BCP is the site of implant placement. The trabecular bone appears to allow more rapid tissue growth than the cortical bone presumably due to the richer vascularity of such structures.

In view of poor vascular supply of periodontal implant sites, NBCP in this study has a high porosity of $85 \%$ and possesses more biodegradable $\beta$-TCP than HA to facilitate its biodegradation. NBCP has a porous structure with a high porosity of $85 \%$. There are abundant micropores in the walls of inter channelled macropores. At 16 weeks of healing, NBCP were absorbed completely and no residual material was found in the implant site[8].

The aim of the experiment was to study the histological reaction of orthodontic force on the periodontal tissues regenerated by nano biphasic calcium phosphate (NBCP) bioceramics in beagle dogs on the basis of previous researches. There was no significant difference in the histological reaction induced by orthodontic tooth movement between the regenerated periodontal tissues and normal tissues.

Based on these findings, we conclude that regenerated periodontal tissues can bear normal orthodontic force. It seems that $\mathrm{NBCP}$, as a promising bioceramic, may provide a new therapy method for the patient who has lost his periodontal tissue and has the demand for orthodontic treatment.

\section{Acknowledgement}

The research was sponsored by the Shandong provincial-level Project for science and technology development on medicine and health (2016WS0588) and the Shandong provincial-level Project for science and technology development on traditional Chinese medicine (2011-100) and the Shandong province natural science Fund (ZR2011HM017).

\section{References}

[1] Reynolds MA, Aichelmann-Reidy ME, Branch-Mays GL, Gunsolley JC (2003) the efficacy of bone replacement grafts in the treatment of periodontal osseous defects. Ann periodontal 8:227-65

[2] Seo BM, Miura M, Gronthos S, et al. Investigation of multipotent postnatal stem cells from human periodontal ligament.Lancet.2004;364:149-155.

[3] Sergey V. Dorozhkin, Biphasic, triphasic and multiphasic calcium orthophosphates,Acta Biomaterialia,2012 (8) 3: 963-977

[4] T Inukai, W Katagiri, R Yoshimi, Novel application of stem cell-derived factors for periodontal regeneration,Biochemical and Biophysical Research Communications,2012 (430) 2: 763-768 
[5] Paul Monsarrat, Jean-Noël Vergnes, Cathy Nabet, Concise Review: Mesenchymal Stromal Cells Used for Periodontal Regeneration: A Systematic Review, Stem Cells Transl Med. 2014(3)6: 768-774

[6] Toshiyuki Yoshida, Kaoru Washio, Takanori Iwata, Current Status and Future Development of Cell Transplantation Therapy for Periodontal Tissue Regeneration,International Journal of Dentistry, 2012 (2012) 1-8

[7] FM Chen, HH Sun, Stem cell-delivery therapeutics for periodontal tissue regeneration, Biomaterials, 2012(33)27: 6320-6344

[8] Lanlei Wang,Han Shi, Yijia Chen, Healing of acute alveolar bone dehiscence following treatment with porous biphasic calcium phosphate in beagle dogs, Clinical Oral Investigations,2011(15)6:983-991

[9] Shangfeng Qi, Lanlei Wang, Efficacy of nano biphasic calcium phosphate in the treatment of osseous defects, Integrated Ferroelectrics, 2011(129)1:196-200

[10]Lanlei Wang,Aizhong Guan, Han Shi, Yangxi Chen, Yunmao Liao, Effect of biphasic calcium phosphate nanocomposite on healing of surgically created alveolar bone defects in beagle dogs,Journal of Physics: Conference Series, 188 (2009) 012049:1-5.

[11] Vassilis Karageorgiou, David Kaplan, Porosity of 3D biomaterial scaffolds and osteogenesis. Biomaterial, 2005; 26: 5474-5491.

[12] Kokubo T, Matsushita T, Takadama H, Kizuki T (2009) Development of bioactive materials based on surface chemistry. J Eur Ceram Soc 29:1267-1274 